\title{
Correction to: Multiple attribute group decision-making based on generalized aggregation operators under linguistic interval-valued Pythagorean fuzzy environment
}

\author{
Rajkumar Verma ${ }^{1}$ (D) Nikunj Agarwal $^{2}$ (D)
}

Published online: 24 December 2021

(C) The Author(s), under exclusive licence to Springer Nature Switzerland AG 2021

\section{Correction to: Granular Computing (2021) https://doi. org/10.1007/s41066-021-00286-y}

In this article the affiliation "Department of Management Control and Information Systems, University of Chile, Av. Diagonal Paraguay 257, 8330015, Santiago, Chile" for author Rajkumar Verma was missing.

The affiliation "Department of Mathematics, JSS Academy of Technical Education, C-20/1, Industrial Area, Sector 62, Noida, UP, 201307, India" for author Nikunj Agarwal was missing.

In Table 2 of this article, few entries in the column headed Operational law were mistakenly listed under a separate column. The corrected Table 2 is given below.

Few lists are aligned incorrectly in the original publication. The corrected texts are given below.

The contributions of this work are as follows:

- Defines four new generalized operational laws between pairs of LIVPFNs based on ATN and ACN with LSF and discusses their properties in detail.

- Some new generalized AOs for LIVPFNs, namely, GALIVPFWA operator, GALIV-PFOWA, and GALIVPFOWAWA operator are proposed.

The original article can be found online at https:// doi.org/10.1007/s41066-021-00286-y.

Rajkumar Verma

rkver83@gmail.com; rverma@fen.uchile.cl

Nikunj Agarwal

nikunjagarwal@jssaten.ac.in

1 Department of Management Control and Information Systems, University of Chile, Av. Diagonal Paraguay 257, 8330015 Santiago, Chile

2 Department of Mathematics, JSS Academy of Technical Education, C-20/1, Industrial Area, Sector 62, Noida, UP 201307, India
- Several mathematical properties of the developed AOs are studied with their special and limiting cases.

- To handle complex realistic decision-making problems, a MAGDM method is formulated based on these AOs to demonstrate the applicability, utility, and flexibility of the developed work.

- The application of the proposed MAGDM method is shown with the help of a real-life numerical example.

- At the end, the advantages and characteristics of proposed method are discussed by comparison analysis.

Definition 3 (Herrera and Martínez 2001) Let $\mathcal{L}=$ $\left\{\ell_{d} \mid d=0,1, \ldots, 2 t\right\}$ be a finite linguistic term set (LTS) with the odd cardinality. Any level $\ell_{d}$ represents a possible linguistic term for a linguistic variable and $t$ is a positive integer. The LTS $\mathcal{L}$ should hold the following properties:

(i) Order relation: If $\ell_{i} \leq \ell_{j} \Leftrightarrow i \leq j$;

(ii) Negation operator: $\operatorname{neg}\left(\ell_{d}\right)=\ell_{2 t-d}$;

(iii) Maximum operator: $\max \left(\ell_{i}, \ell_{j}\right)=\ell_{i} \Leftrightarrow i \geq j$;

(iv) Minimum operator: $\min \left(\ell_{i}, \ell_{j}\right)=\ell_{i} \Leftrightarrow i \leq j$.

For example, a set of seven linguistic terms can be represented as:

$$
\mathcal{L}=\left\{\begin{array}{l}
\ell_{0}=\operatorname{none}(\mathrm{N}), \ell_{1}=\operatorname{very} \operatorname{low}(\mathrm{VL}), \\
\ell_{2}=\operatorname{low}(\mathrm{L}), \ell_{3}=\operatorname{median}(\mathrm{M}), \ell_{4}=\operatorname{high}(\mathrm{H}), \\
\ell_{5}=\operatorname{very} \operatorname{high}(\mathrm{VH}), \ell_{6}=\operatorname{perfect}(\mathrm{P})
\end{array}\right\} .
$$

Few mathematical expressions are incorrect, and the correct equations are provided below. 
Table 2 The values of Archimedean operational laws taking different forms of $\mathcal{G}(\varepsilon)$

\begin{tabular}{|c|c|c|c|}
\hline \multirow[t]{2}{*}{ Operational law } & \multicolumn{3}{|l|}{ LSF } \\
\hline & $\mathfrak{I}^{*}=\mathfrak{I}_{1}^{*}$ & $\begin{array}{l}\mathfrak{I}^{*}=\mathfrak{I}_{2}^{*} \\
(\varrho=1.5)\end{array}$ & $\begin{array}{c}\mathfrak{I}^{*}=\mathfrak{I}_{3}^{*} \\
(\rho=\tau=0.8)\end{array}$ \\
\hline \multicolumn{4}{|l|}{ Algebraic } \\
\hline $\mathfrak{I}_{1} \underset{A}{\stackrel{\star}{\oplus} \mathfrak{I}_{2}}$ & $\left\langle\left[\ell_{3.5264}, \ell_{4.7696}\right],\left[\ell_{0.1248}, \ell_{2.2504}\right]\right\rangle$ & $\left\langle\left[\ell_{4.6590}, \ell_{5.7574}\right],\left[\ell_{0.1768}, \ell_{1.5203}\right]\right\rangle$ & $\left\langle\left[\ell_{3.4007}, \ell_{4.3928}\right],\left[\ell_{0.1057}, \ell_{2.4334}\right]\right\rangle$ \\
\hline$\underset{A}{\mathfrak{J}_{1} \stackrel{\star}{\otimes} \mathfrak{I}_{2}}$ & $\left\langle\left[\ell_{0.7504}, \ell_{1.5000}\right],\left[\ell_{1.4088}, \ell_{6.3200}\right]\right\rangle$ & $\left\langle\left[\ell_{0.6887}, \ell_{1.5483}\right],\left[\ell_{1.0699}, \ell_{6.6214}\right]\right\rangle$ & $\left\langle\left[\ell_{0.6998}, \ell_{1.5988}\right],\left[\ell_{1.3930}, \ell_{6.2353}\right]\right\rangle$ \\
\hline $3_{A}^{\star} \mathfrak{I}$ & $\left\langle\left[\ell_{6.6144}, \ell_{7.4280}\right],\left[\ell_{0.0312}, \ell_{0.5000}\right]\right\rangle$ & $\left\langle\left[\ell_{7.1967}, \ell_{7.4885}\right],\left[\ell_{0.0577}, \ell_{0.2607}\right]\right\rangle$ & $\left\langle\left[\ell_{6.3507}, \ell_{7.5093}\right],\left[\ell_{0.2010}, \ell_{0.6149}\right]\right\rangle$ \\
\hline $\mathfrak{I}_{A}^{\star} 3$ & $\left\langle\left[\ell_{0.5000}, \ell_{1.2208}\right],\left[\ell_{3.8160}, \ell_{6.6144}\right]\right\rangle$ & $\left\langle\left[\ell_{0.2607}, \ell_{0.4286}\right],\left[\ell_{5.8012}, \ell_{7.1967}\right]\right\rangle$ & $\left\langle\left[\ell_{0.6149}, \ell_{1.8472}\right],\left[\ell_{3.5395}, \ell_{6.3507}\right]\right\rangle$ \\
\hline \multicolumn{4}{|l|}{ Einstein } \\
\hline $\mathfrak{I}_{1} \underset{E}{\stackrel{\star}{\oplus} \mathfrak{I}_{2}}$ & $\left\langle\left[\ell_{3.5896}, \ell_{4.9144}\right],\left[\ell_{0.0888}, \ell_{1.9184}\right]\right\rangle$ & $\left\langle\left[\ell_{4.8748}, \ell_{5.9619}\right],\left[\ell_{0.1267}, \ell_{1.1811}\right]\right\rangle$ & $\left\langle\left[\ell_{3.4381}, \ell_{4.4925}\right],\left[\ell_{0.0749}, \ell_{2.1321}\right]\right\rangle$ \\
\hline 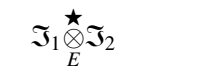 & $\left\langle\left[\ell_{0.5584}, \ell_{1.1696}\right],\left[\ell_{1.4144}, \ell_{6.4576}\right]\right\rangle$ & $\left\langle\left[\ell_{0.5086}, \ell_{0.8022}\right],\left[\ell_{1.5707}, \ell_{6.7790}\right]\right\rangle$ & $\left\langle\left[\ell_{0.5179}, \ell_{1.2528}\right],\left[\ell_{1.3967}, \ell_{6.3552}\right]\right\rangle$ \\
\hline $3_{E}^{\star \star} \mathfrak{I}$ & $\left\langle\left[\ell_{7.0224}, \ell_{7.7104}\right],\left[\ell_{0.0120}, \ell_{0.2312}\right]\right\rangle$ & $\left\langle\left[\ell_{7.4616}, \ell_{7.7021}\right],\left[\ell_{0.0227}, \ell_{0.1171}\right]\right\rangle$ & $\left\langle\left[\ell_{6.8179}, \ell_{7.7792}\right],\left[\ell_{0.0080}, \ell_{0.2869}\right]\right\rangle$ \\
\hline $\mathfrak{I}_{E}^{\star} 3$ & $\left\langle\left[\ell_{0.2312}, \ell_{0.6656}\right],\left[\ell_{3.9616}, \ell_{7.0224}\right]\right\rangle$ & $\left\langle\left[\ell_{0.1171}, \ell_{0.2042}\right],\left[\ell_{6.1322}, \ell_{7.4616}\right]\right\rangle$ & $\left\langle\left[\ell_{0.2869}, \ell_{1.1008}\right],\left[\ell_{3.6166}, \ell_{6.8179}\right]\right\rangle$ \\
\hline \multicolumn{4}{|l|}{ Hamacher $(\gamma=3)$} \\
\hline $\mathfrak{I}_{1} \stackrel{\oplus}{\oplus}_{\mathfrak{I}_{2}}$ & $\left\langle\left[\ell_{3.6504}, \ell_{5.0456}\right],\left[\ell_{0.0728}, \ell_{1.7000}\right]\right\rangle$ & $\left\langle\left[\ell_{5.0602}, \ell_{6.1297}\right],\left[\ell_{0.1035}, \ell_{0.9935}\right]\right\rangle$ & $\left\langle\left[\ell_{3.4737}, \ell_{4.5890}\right],\left[\ell_{0.0609}, \ell_{1.9211}\right]\right\rangle$ \\
\hline 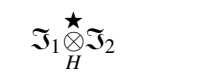 & $\left\langle\left[\ell_{0.4640}, \ell_{0.9912}\right],\left[\ell_{1.4192}, \ell_{6.5744}\right]\right\rangle$ & $\left\langle\left[\ell_{0.4197}, \ell_{0.6657}\right],\left[\ell_{1.5917}, \ell_{6.9037}\right]\right\rangle$ & $\left\langle\left[\ell_{0.4292}, \ell_{1.0651}\right],\left[\ell_{1.3995}, \ell_{6.4561}\right]\right\rangle$ \\
\hline $3_{H}^{\star \star} \mathfrak{I}$ & $\left\langle\left[\ell_{7.3032}, \ell_{7.8384}\right],\left[\ell_{0.0064}, \ell_{0.1384}\right]\right\rangle$ & $\left\langle\left[\ell_{7.6286}, \ell_{7.8155}\right],\left[\ell_{0.0131}, \ell_{0.0694}\right]\right\rangle$ & $\left\langle\left[\ell_{7.1489}, \ell_{7.8863}\right],\left[\ell_{0.0040}, \ell_{0.1722}\right]\right\rangle$ \\
\hline $\mathfrak{I}_{H}^{\star} 3$ & $\left\langle\left[\ell_{0.1384}, \ell_{0.4288}\right],\left[\ell_{4.1072}, \ell_{7.3032}\right]\right\rangle$ & $\left\langle\left[\ell_{0.0694}, \ell_{0.1246}\right],\left[\ell_{6.4052}, \ell_{7.6286}\right]\right\rangle$ & $\left\langle\left[\ell_{0.1722}, \ell_{0.7402}\right],\left[\ell_{3.6914}, \ell_{7.1489}\right]\right\rangle$ \\
\hline \multicolumn{4}{|l|}{ Frank $(\delta=3)$} \\
\hline $\mathfrak{I}_{1} \underset{F}{\stackrel{\star}{\oplus}} \mathfrak{I}_{2}$ & $\left\langle\left[\ell_{3.5576}, \ell_{4.8456}\right],\left[\ell_{0.0936}, \ell_{2.0048}\right]\right\rangle$ & $\left\langle\left[\ell_{4.7715}, \ell_{5.8697}\right],\left[\ell_{0.1330}, \ell_{1.2583}\right]\right\rangle$ & $\left\langle\left[\ell_{3.4185}, \ell_{4.4444}\right],\left[\ell_{0.0788}, \ell_{2.2141}\right]\right\rangle$ \\
\hline$\underset{F}{\mathfrak{I}_{1} \stackrel{\star}{\stackrel{\mathfrak{I}}{\mathfrak{I}}} 2}$ & $\left\langle\left[\ell_{0.5872}, \ell_{1.2320}\right],\left[\ell_{1.4112}, \ell_{6.4016}\right]\right\rangle$ & $\left\langle\left[\ell_{0.5384}, \ell_{0.8537}\right],\left[\ell_{1.5587}, \ell_{6.7138}\right]\right\rangle$ & $\left\langle\left[\ell_{0.5442}, \ell_{1.3166}\right],\left[\ell_{1.3949}, \ell_{6.3067}\right]\right\rangle$ \\
\hline $3 \underset{F}{*} \mathfrak{I}$ & $\left\langle\left[\ell_{6.8680}, \ell_{7.6344}\right],\left[\ell_{0.0136}, \ell_{0.2696}\right]\right\rangle$ & $\left\langle\left[\ell_{7.3647}, \ell_{7.6341}\right],\left[\ell_{0.0267}, \ell_{0.1371}\right]\right\rangle$ & $\left\langle\left[\ell_{6.6391}, \ell_{7.7161}\right],\left[\ell_{0.0094}, \ell_{0.3341}\right]\right\rangle$ \\
\hline$\stackrel{\Im}{\star} 3$ & $\left\langle\left[\ell_{0.2696}, \ell_{0.7728}\right],\left[\ell_{3.8864}, \ell_{6.8680}\right]\right\rangle$ & $\left\langle\left[\ell_{0.1371}, \ell_{0.2397}\right],\left[\ell_{5.9781}, \ell_{7.3647}\right]\right\rangle$ & $\left\langle\left[\ell_{0.3341}, \ell_{1.2667}\right],\left[\ell_{3.5768}, \ell_{6.6391}\right]\right\rangle$ \\
\hline
\end{tabular}

(iii) $\quad \underset{E}{\lambda *} \mathfrak{J}=\left\langle\left[\mathfrak{I}^{*-1}\left(\sqrt{\left(\frac{\left(1+\left(\mu^{L}\right)^{2}\right)^{\lambda}-\left(1-\left(\mu^{L}\right)^{2}\right)^{\lambda}}{\left(1+\left(\mu^{L}\right)^{2}\right)^{\lambda}+\left(1-\left(\mu^{L}\right)^{2}\right)^{\lambda}}\right)}\right)\right.\right.$,

$$
\begin{aligned}
& \left.\mathfrak{I}^{*-1}\left(\sqrt{\left(\frac{\left(1+\left(\mu^{U}\right)^{2}\right)^{\lambda}-\left(1-\left(\mu^{U}\right)^{2}\right)^{\lambda}}{\left(1+\left(\mu^{U}\right)^{2}\right)^{\lambda}+\left(1-\left(\mu^{U}\right)^{2}\right)^{\lambda}}\right)}\right)\right], \\
& {\left[\mathfrak{I}^{*-1}\left(\frac{\sqrt{2}\left(v^{L}\right)^{\lambda}}{\sqrt{\left(2-\left(v^{L}\right)^{2}\right)^{\lambda}+\left(\left(v^{L}\right)^{2}\right)^{\lambda}}}\right),\right.} \\
& \left.\left.\mathfrak{I}^{*-1}\left(\frac{\sqrt{2}\left(v^{U}\right)^{\lambda}}{\sqrt{\left(2-\left(v^{U}\right)^{2}\right)^{\lambda}+\left(\left(v^{U}\right)^{2}\right)^{\lambda}}}\right)\right]\right\rangle, \lambda>0 ;
\end{aligned}
$$

$$
\begin{aligned}
& \left\langle\left[\ell_{3.4007}, \ell_{4.3928}\right],\left[\ell_{0.1057}, \ell_{2.4334}\right]\right\rangle \\
& \left\langle\left[\ell_{0.6998}, \ell_{1.5988}\right],\left[\ell_{1.3930}, \ell_{6.2353}\right]\right\rangle \\
& \left\langle\left[\ell_{6.3507}, \ell_{7.5093}\right],\left[\ell_{0.2010}, \ell_{0.6149}\right]\right\rangle \\
& \left\langle\left[\ell_{0.6149}, \ell_{1.8472}\right],\left[\ell_{3.5395}, \ell_{6.3507}\right]\right\rangle
\end{aligned}
$$

$\left\langle\left[\ell_{3.4381}, \ell_{4.4925}\right],\left[\ell_{0.0749}, \ell_{2.1321}\right]\right\rangle$

$\left\langle\left[\ell_{0.5179}, \ell_{1.2528}\right],\left[\ell_{1.3967}, \ell_{6.3552}\right]\right\rangle$

$\left\langle\left[\ell_{6.8179}, \ell_{7.7792}\right],\left[\ell_{0.0080}, \ell_{0.2869}\right]\right\rangle$

$\left\langle\left[\ell_{0.2869}, \ell_{1.1008}\right],\left[\ell_{3.6166}, \ell_{6.8179}\right]\right\rangle$

$\left\langle\left[\ell_{3.4737}, \ell_{4.5890}\right],\left[\ell_{0.0609}, \ell_{1.9211}\right]\right\rangle$

$\left\langle\left[\ell_{0.4292}, \ell_{1.0651}\right],\left[\ell_{1.3995}, \ell_{6.4561}\right]\right\rangle$

$\left\langle\left[\ell_{7.1489}, \ell_{7.8863}\right],\left[\ell_{0.0040}, \ell_{0.1722}\right]\right\rangle$

$\left\langle\left[\ell_{0.1722}, \ell_{0.7402}\right],\left[\ell_{3.6914}, \ell_{7.1489}\right]\right\rangle$

$\left\langle\left[\ell_{3.4185}, \ell_{4.4444}\right],\left[\ell_{0.0788}, \ell_{2.2141}\right]\right\rangle$

$\left\langle\left[\ell_{0.5442}, \ell_{1.3166}\right],\left[\ell_{1.3949}, \ell_{6.3067}\right]\right\rangle$

$\left\langle\left[\ell_{6.6391}, \ell_{7.7161}\right],\left[\ell_{0.0094}, \ell_{0.3341}\right]\right\rangle$

$\left\langle\left[\ell_{0.3341}, \ell_{1.2667}\right],\left[\ell_{3.5768}, \ell_{6.6391}\right]\right\rangle$ (iv)

$$
\begin{aligned}
& \mathfrak{I}_{E}^{\star} \lambda=\left\langle\left[\mathfrak{I}^{*-1}\left(\frac{\sqrt{2}\left(\mu^{L}\right)^{\lambda}}{\sqrt{\left(2-\left(\mu^{L}\right)^{2}\right)^{\lambda}+\left(\left(\mu^{L}\right)^{2}\right)^{\lambda}}}\right),\right.\right. \\
& \left.\mathfrak{I}^{*-1}\left(\frac{\sqrt{2}\left(\mu^{U}\right)^{\lambda}}{\sqrt{\left(2-\left(\mu^{U}\right)^{2}\right)^{\lambda}+\left(\left(\mu^{U}\right)^{2}\right)^{\lambda}}}\right)\right], \\
& {\left[\mathfrak{I}^{*-1}\left(\sqrt{\left(\frac{\left(1+\left(v^{L}\right)^{2}\right)^{\lambda}-\left(1-\left(v^{L}\right)^{2}\right)^{\lambda}}{\left(1+\left(v^{L}\right)^{2}\right)^{\lambda}+\left(1-\left(v^{L}\right)^{2}\right)^{\lambda}}\right)}\right),\right.} \\
& \left.\left.\mathfrak{I}^{*-1}\left(\sqrt{\left(\frac{\left(1+\left(v^{U}\right)^{2}\right)^{\lambda}-\left(1-\left(v^{U}\right)^{2}\right)^{\lambda}}{\left(1+\left(v^{U}\right)^{2}\right)^{\lambda}+\left(1-\left(v^{U}\right)^{2}\right)^{\lambda}}\right)}\right)\right]\right\rangle, \lambda>0 .
\end{aligned}
$$


(iii) $\quad \underset{H}{\lambda *} \mathfrak{\star} \mathfrak{I}=\left\langle\left[\mathfrak{I}^{*-1}\left(\sqrt{\left(\frac{\left(1+(\gamma-1)\left(\mu^{L}\right)^{2}\right)^{\lambda}-\left(1-\left(\mu^{L}\right)^{2}\right)^{\lambda}}{\left(1+(\gamma-1)\left(\mu^{L}\right)^{2}\right)^{\lambda}+(\gamma-1)\left(1-\left(\mu^{L}\right)^{2}\right)^{\lambda}}\right.}\right)\right)\right.$,

(iii)

$$
\begin{aligned}
& \underset{F}{\lambda \underset{*}{*} \mathfrak{I}}=\left\langle\left[\mathfrak{I}^{*-1}\left(\sqrt{\left(1-\log _{\delta}\left(1+\frac{\left(\delta^{1-\left(\mu^{L}\right)^{2}}-1\right)^{\lambda}}{(\delta-1)^{\lambda-1}}\right)\right.}\right)\right),\right. \\
& \left.\mathfrak{I}^{*-1}\left(\sqrt{\left(1-\log _{\delta}\left(1+\frac{\left(\delta^{1-\left(\mu^{U}\right)^{2}-1}\right)^{\lambda}}{(\delta-1)^{\lambda-1}}\right)\right)}\right)\right] \text {, } \\
& {\left[\mathfrak{I}^{*-1}\left(\sqrt{\left(\log _{\delta}\left(1+\frac{\left(\delta^{\left({ }^{(L}\right)^{2}}-1\right)^{\lambda}}{(\delta-1)^{\lambda-1}}\right)\right.}\right)\right),} \\
& \left.\left.\mathfrak{T}^{*-1}\left(\sqrt{\left(\log _{\delta}\left(1+\frac{\left(\delta^{\left(v^{U}\right)^{2}}-1\right)^{\lambda}}{(\delta-1)^{\lambda-1}}\right)\right.}\right)\right)\right] ;
\end{aligned}
$$$$
\left.\mathfrak{I}^{*-1}\left(\sqrt{\left(\frac{\left(1+(\gamma-1)\left(\mu^{U}\right)^{2}\right)^{\lambda}-\left(1-\left(\mu^{U}\right)^{2}\right)^{\lambda}}{\left(1+(\gamma-1)\left(\mu^{U}\right)^{2}\right)^{\lambda}+(\gamma-1)\left(1-\left(\mu^{U}\right)^{2}\right)^{\lambda}}\right)}\right)\right],
$$$$
\left[\mathfrak{I}^{*-1}\left(\frac{\sqrt{\gamma}\left(v^{L}\right)^{\lambda}}{\sqrt{\left(1+(\gamma-1)\left(1-\nu^{L}\right)^{2}\right)^{\lambda}+(\gamma-1)\left(v^{L}\right)^{2 \lambda}}}\right),\right.
$$$$
\left.\left.\mathfrak{I}^{*-1}\left(\frac{\sqrt{\gamma}\left(v^{U}\right)^{\lambda}}{\sqrt{\left(1+(\gamma-1)\left(1-v^{U}\right)^{2}\right)^{\lambda}+(\gamma-1)\left(v^{U}\right)^{2 \lambda}}}\right)\right]\right\rangle, \lambda>0 ;
$$

(iv) $\quad \underset{H}{\mathfrak{I}_{H}^{\star}} \lambda=\left\langle\left[\mathfrak{I}^{*-1}\left(\frac{\sqrt{\gamma}\left(\mu^{L}\right)^{\lambda}}{\sqrt{\left(1+(\gamma-1)\left(1-\mu^{L}\right)^{2}\right)^{\lambda}+(\gamma-1)\left(\mu^{L}\right)^{2 \lambda}}}\right)\right.\right.$,

$$
\begin{gathered}
\left.\mathfrak{I}^{*-1}\left(\frac{\sqrt{\gamma}\left(\mu^{U}\right)^{\lambda}}{\sqrt{\left(1+(\gamma-1)\left(1-\mu^{U}\right)^{2}\right)^{\lambda}+(\gamma-1)\left(\mu^{U}\right)^{2 \lambda}}}\right)\right], \\
{\left[\mathfrak{I}^{*-1}\left(\sqrt{\left(\frac{\left(1+(\gamma-1)\left(v^{L}\right)^{2}\right)^{\lambda}-\left(1-\left(v^{L}\right)^{2}\right)^{\lambda}}{\left(1+(\gamma-1)\left(v^{L}\right)^{2}\right)^{\lambda}+(\gamma-1)\left(1-\left(v^{L}\right)^{2}\right)^{\lambda}}\right)}\right),\right.} \\
\left.\left.\mathfrak{I}^{*-1}\left(\sqrt{\left(\frac{\left(1+(\gamma-1)\left(v^{U}\right)^{2}\right)^{\lambda}-\left(1-\left(v^{U}\right)^{2}\right)^{\lambda}}{\left(1+(\gamma-1)\left(v^{U}\right)^{2}\right)^{\lambda}+(\gamma-1)\left(1-\left(v^{U}\right)^{2}\right)^{\lambda}}\right)}\right)\right]\right\rangle, \lambda>0 .
\end{gathered}
$$

The original article has been corrected. 